\title{
Vascular gene transfer and drug delivery in vitro using low-frequency ultrasound and microbubbles
}

\author{
Hong YANG ${ }^{1, \#}$, Zhong-hua LIU 2, \#, Yi-yao LIU ${ }^{1, *}$, Chang-chun LOU ${ }^{1}$, Zheng-long REN ${ }^{1}$, Hirokazu MIYOSHI ${ }^{3}$ \\ ${ }^{1}$ Department of Biophysics, School of Life Science and Technology, University of Electronic Science and Technology of China, Chengdu \\ 610054, China; ${ }^{2}$ School of Science, Southwest Petroleum University, Chengdu 610500, China; ${ }^{3}$ Radioisotope Research Center, Univer- \\ sity of Tokushima, Kuramoto-cho 3-18-15, Tokushima 770-8503, Japan
}

\begin{abstract}
Aim: To determine the effects of ultrasound exposure in combination with a microbubble contrast agent (SonoVue) on the cellular uptake and delivery of drugs/genes into human umbilical vein endothelial cells (HUVECs) as well as their biological effects on migration.

Methods: HUVECs in suspension were exposed to pulsed ultrasound with a $10 \%$ duty cycle in combination with various concentrations of a microbubble contrast agent (SonoVue) using a digital sonifier at a frequency of $20 \mathrm{kHz}$ and an intensity of $3.77 \mathrm{~W} / \mathrm{cm}^{2}$ on the surface of a horn tip. Cell culture inserts were used to determine the cell migration ability.

Results: Exposure to pulsed ultrasound resulted in enhanced green fluorescent protein (EGFP) gene transfection efficiencies ranging from $0.2 \%$ to $2 \%$. The transfection efficiency of HUVECs was approximately 3 -fold higher in the presence of SonoVue than in its absence at the effective exposure time of $6 \mathrm{~s}$. For drug delivery to HUVECs using ultrasound, the delivery efficiencies of a lowmolecular-weight model drug (TO-PRO ${ }^{\circledR}-1, \mathrm{M}_{\mathrm{W}}$ 645.38) were significantly higher when compared to drug delivery without ultrasound, with a maximum efficiency of approximately 34\%. However, the delivery efficiencies of a high-molecular-weight model drug (DextranRhodamine $B, M_{w} 70000$ ) were low, with a maximum delivery efficiency of nearly $0.5 \%$, and gene transfection results were similarly poor. The migration ability of HUVECs exposed to ultrasound was also lower than that of the control (no exposure).

Conclusion: The use of low-frequency and low-energy ultrasound in combination with microbubbles could be a potent physical method of increasing drug/gene delivery efficiency. This technique is a promising nonviral approach that can be used in cardiovascular disease therapy.
\end{abstract}

Keywords: ultrasound exposure; human umbilical vein endothelial cells; microbubble; gene transfection; drug delivery; cell migration

Acta Pharmacologica Sinica (2010) 31: 515-522; doi: 10.1038/aps.2010.21; published online 29 Mar 2010

\section{Introduction}

Gene therapy and drug delivery are promising tools for the treatment of several conditions, such as cancer and cardiovascular diseases $^{[1,2]}$. However, current clinical applications are hampered by the absence of safe and efficient systems for local gene/drug delivery to specific tissues or organs. For gene therapy, nonviral gene delivery can be performed by the direct injection of DNA, but such approaches are generally associated with low transfection efficiencies and only transient expression of the gene product. Viral vectors significantly increase transfection efficiency because of the specific viral machinery that has evolved to introduce foreign DNA into

\footnotetext{
\# These authors contributed equally to this work.

* To whom correspondence should be addressed.

E-mail liuyiyao@hotmail.com

Received 2009-10-03 Accepted 2010-01-29
}

mammalian cells. However, viral proteins elicit an unwanted immune response within the targeted hosts or tissues ${ }^{[3]}$.

For many decades, ultrasound technology has been widely used for diagnostic imaging in clinical fields without any significant adverse side effects. In recent years, there has been intense interest in developing an efficient, simple way to deliver DNA or drugs into cells ${ }^{[4]}$. It has been shown that sonication (ultrasound) can alter the transient permeability of plasma membranes to facilitate uptake ${ }^{[5]}$. Compared with other direct DNA delivery methods, such as particle gun bombardment, electroporation, and microinjection, sonication may be simpler to carry out. Sonication, however, can cause cell damage or even rupture. If sonication is used to facilitate uptake, it is important to optimize the conditions or parameters without causing cell damage. Mild ultrasound irradiation has proved to be an efficient method of transfecting genes into animal cells and tissues in vitro and in vivo ${ }^{[6,7]}$. 
Encapsulated gas microbubbles (usually $2-8 \mu \mathrm{m}$ in diameter) have been used as ultrasound contrast agents in medical ultrasound imaging for several decades. Recently, ultrasoundinduced microbubble destruction has been proposed as a new technique for the local delivery of genes and drugs to specific target tissues, including the heart ${ }^{[8-10]}$. Ultrasound can cause a transient, nonlethal perforation of the capillary and cell membranes because of cavitation effects and thereby improve transfection and drug delivery efficiencies. In many studies, ultrasound-enhanced gene/drug delivery techniques combined with microbubbles encasing an expression vector have been shown to enhance gene transfection and drug delivery ${ }^{[10,11]}$. These studies focused mainly on cancer and the vascular system. The cavitation of microbubbles under an ultrasound field will lead to an increase in microvascular permeability and induce small gaps between capillary endothelial cells due to the cavitation energy, which will enable drugs to leak from the vessel and reach the target location ${ }^{[9,11,12]}$. Recently, Ohta et al used microbubble-enhanced sonoporation to achieve ectopic and transient gene expression in several embryonic organs, including embryonic chick limb bud mesenchymes $^{[13]}$. Recently, ultrasound has also been used as an external trigger for the controlled release of drugs ${ }^{[14,15]}$. These studies showed that ultrasound is one of the most promising external triggers for pulsatile delivery, and the release rate of the incorporated drug can be altered by external application of ultrasound radiation ${ }^{[15]}$. Although ultrasound is widely applied in gene transfection and drug delivery, different ultrasound exposure modalities or setups will result in different delivery efficiencies of DNA or drugs ${ }^{[3,16,17]}$.

In this research, gene transfection and drug delivery by sonoporation were examined using cultured vascular endothelial cells (HUVECs) with a microbubble contrast agent (SonoVue). Various parameters and experimental conditions for sonoporation to introduce exogenous molecules (DNA and drugs) into HUVECs were used and discussed. The feasibility and mechanisms of gene and drug delivery to HUVECs were tested in our exposure setup. The cytoskeleton arrangement and migration ability of HUVECs after ultrasound exposure were also investigated.

\section{Materials and methods Reagents}

Cell culture medium RPMI 1640, HAT (hypoxanthine, aminopterin, and thymidine) supplement, and newborn calf serum (NCS) were purchased from Gibco (Grand Island, NY, USA). Dimethyl sulfoxide (DMSO), 3-(4,5-dimethylthiazol-2-yl)3,5-diphenyltetrazolium (MTT), and 2-[4-(2-hydroxyethyl)1-piperazine]ethanesulfonic acid (HEPES) were provided by Amresco (Cleveland, OH, USA). Trypsin, penicillin and streptomycin were obtained from Sigma-Aldrich (St Louis, MO, USA). SonoVue microbubbles were purchased from BRACCO (Milan, Italy). TO-PRO ${ }^{\circledR}-1$ and Dextran-Rhodamine B were obtained from Molecular Probes (Invitrogen). All other chemicals were of analytical grade and used without further purification.

\section{Cell culture}

The HUVEC line EA Hy926 (passages 5-8) was obtained from the Institute of Biomedical Engineering of the Western China Medical College (Sichuan, China). This cell line, a hybridoma of HUVECs and the human epithelial cell line A549, has been shown to retain a number of properties of endothelial cells, including the production of human factor VIII-related antigen. The cells were cultured in RPMI 1640 containing $20 \mathrm{mmol} / \mathrm{L}$ HEPES, $10 \% \mathrm{NCS}, 2 \% \mathrm{NaHCO}_{3}, 100 \mathrm{U} / \mathrm{mL}$ penicillin, 100 $\mathrm{mg} / \mathrm{mL}$ streptomycin, and $2 \%$ HAT supplement at a temperature of $37^{\circ} \mathrm{C}$ in a humidified $5 \% \mathrm{CO}_{2}$ atmosphere. Confluent cells were trypsinized and seeded at a density of $1 \times 10^{5}$ cells/ well $(1 \mathrm{~mL})$ in 12-well plates (Corning, NY, USA). The culture medium was removed when the cells reached $70-80 \%$ confluence, and $2 \mathrm{~mL}$ of new culture medium containing enhanced green fluorescent protein plasmid (pEGFP-N1, containing an EGFP reporter gene) or model drugs (TO-PRO ${ }^{\circledR}-1$ or DextranRhodamine B) were added to the wells. The samples were then used for ultrasound exposure experiments.

\section{Ultrasound exposure}

Ultrasound was generated using a $20-\mathrm{kHz}$ digital sonifier S-250D (Branson Ultrasonics, Danbury, CT, USA) at a power of $3 \mathrm{~W}$ with an intensity of $3.77 \mathrm{~W} / \mathrm{cm}^{2}$ on the surface of a tapered microtip with a diameter of $12.64 \mathrm{~mm}$ (Figure 1). The vibration amplitude was $9 \%$, and the effective exposure time ranged from 0 to $15 \mathrm{~s}$ (total exposure time $0-150 \mathrm{~s}$ ) with a $10 \%$ duty cycle (ultrasound exposure: on for $0.1 \mathrm{~s}$ and off for $0.9 \mathrm{~s}$ ). The pEGFP-N1 plasmid (or model drug), with or without SonoVue, was added to the cells that were exposed to ultrasound. The control samples received either pEGFP-N1 plasmid (or model drug) only or pEGFP-N1 plasmid plus SonoVue without ultrasound exposure. The exposure experiments were carried out on HUVEC monolayer cultures in 12-well plates.

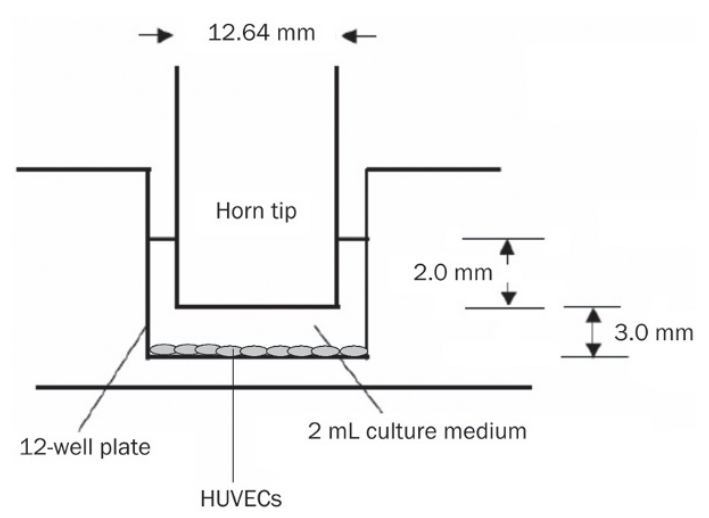

Figure 1. Schematic ultrasound exposure system with digital sonifier at $20 \mathrm{kHz}$ for HUVECs in suspension.

\section{Cell viability assay}

Cell viability was determined by the MTT colorimetric assay. This assay depends on the ability of cells to metabolize a 
yellow tetrazolium salt, MTT bromide dye, to a dark-blue formazan product. Cells $\left(1 \times 10^{4}\right)$ were suspended in $200 \mu \mathrm{L}$ of culture medium and seeded into 96-well plates and incubated for $24 \mathrm{~h}$ at $37^{\circ} \mathrm{C}$ in a $5 \% \mathrm{CO}_{2}$ incubator. Then $20 \mu \mathrm{L}$ of MTT $(0.5 \mathrm{mg} / \mathrm{mL})$ was added into the wells, followed by further incubation for $4 \mathrm{~h}$ at $37^{\circ} \mathrm{C}$. After the supernatants were removed, $150 \mu \mathrm{L}$ of DMSO was added to dissolve the formazan crystals formed. Absorbance was measured colorimetrically at $570 \mathrm{~nm}$. Cell viability was calculated as the percentage of the absorbance of treatment groups relative to that of the control.

\section{Gene/drug delivery assay in vitro}

Cells cultured $24 \mathrm{~h}$ after exposure were washed twice in phosphate-buffered saline (PBS), after which the qualitative analysis of EGFP gene expression or model drug delivery into the cells was observed under an inverted fluorescence microscope (TE-2000U, Nikon, Japan). Flow cytometry analysis was used to quantify the efficiencies of gene transfection and drug delivery into the HUVECs. Samples were analyzed on a FACSCalibur machine (Becton Dickinson, Franklin Lakes, NJ, USA) for fluorescence, and 30000 events were captured for each sample.

\section{Cell migration assay}

The HUVEC migration experimental system utilized Millicell ${ }^{\circledR}$ cell culture inserts (Millipore, Billerica, MA, USA) containing PET membranes with a pore size of $8 \mu \mathrm{m}$. Twelve-well plates were used for the HUVEC migration after ultrasound exposure $^{[18]}$. Each well contained $1.5 \mathrm{~mL}$ of RPMI 1640 culture medium, and $500 \mu \mathrm{L}$ of the exposed cell suspension was added to each insert. The cells were cultured for 2 to $12 \mathrm{~h}$ in a $5 \% \mathrm{CO}_{2}$ incubator at $37{ }^{\circ} \mathrm{C}$. The culture medium was removed, and cells on the PET membranes that had not migrated were scraped off using a cotton stick. The cells that migrated through the pores (determined by adhesion to the outer side of the PET membrane) were fixed for 30 min using a mixed solution $(5 \mathrm{~mL})$ of acetic acid and methyl alcohol (1:3, $v / v)$ after the inserts were washed twice with PBS. The PET membranes were taken out using a scalpel and dyed for 10-15 min with Giemsa solution $(0.9 \%)$ on a glass slice. The excess Giemsa solution was washed with Milli-Q water three times. The numbers of dark blue migrated cells were quantified by cell counting under an inverted microscope from more than ten random microscopic fields (magnification 60×) for each sample.

\section{Presentation of data and statistical analysis}

All data are presented as mean \pm standard deviation (SD). The significance of any differences between experimental groups was assessed using two-tailed Student's t-test. Differences between groups were considered significant at $P<0.05$ or $P<0.01$.

\section{Results and discussion}

Effects of ultrasound and microbubbles on HUVEC viability

The numbers of viable HUVECs decreased linearly with ultra- sound exposure time, as shown in Figure 2A, but the viability was still more than $75 \%$ even at the effective exposure time of $12 \mathrm{~s}$. The mechanical and physical forces of the ultrasound exposure will destroy the cell if the intensity and exposure time are beyond the tolerance limit of the culture and can even induce cell lysis leading to death ${ }^{[19,20]}$. Our data also indicate that the SonoVue microbubbles had a minimal effect on the viability of HUVECs (Figure 2B) as it was measured at more than $90 \%$ even at high concentrations $(40 \%, v / v)$. For a SonoVue concentration of $10 \%(v / v)$, the cell viability observed was very similar to that of the control $(0 \%, v / v)$, indicating that $10 \%$ SonoVue microbubble treatment has almost no effect on the viability of HUVECs. Thus, this concentration was used in the succeeding experiments. In previous studies, microbubbles exhibited side effects when injected into patients ${ }^{[21-23]}$. Our experiments demonstrated that the side effects of SonoVue microbubbles were not as evident as those of other reported ultrasound contrast media. It was also demonstrated that SonoVue is an ideal microbubble for gene/drug delivery.
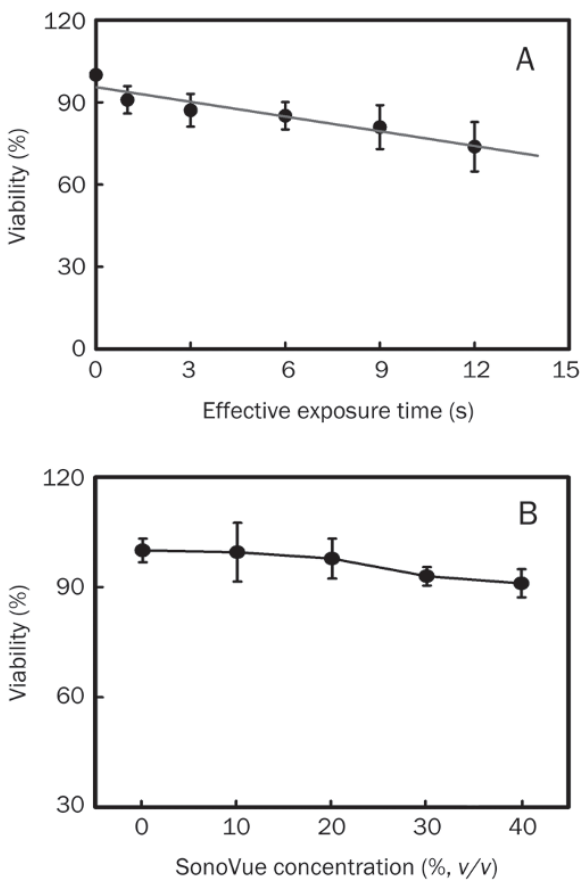

Figure 2. (A) Cell viability versus effective ultrasound exposure time with $10 \%$ duty cycle in the presence of $10 \%(\mathrm{~V} / \mathrm{V})$ SonoVue microbubbles. (B) Cell viability of HUVECs versus various SonoVue concentrations without ultrasound exposure. Each point is the mean of three independent experimental data.

\section{Gene transfer into HUVECs using ultrasound and microbubbles}

After ultrasound exposure, the cells that expressed EGFP were observed under a fluorescence microscope. The EGFP-N1 plasmid DNA was successfully transferred into HUVECs, as shown in Figure 3C. The transfection efficiencies, defined as the ratio of the transfected cells to the total cell number, first 

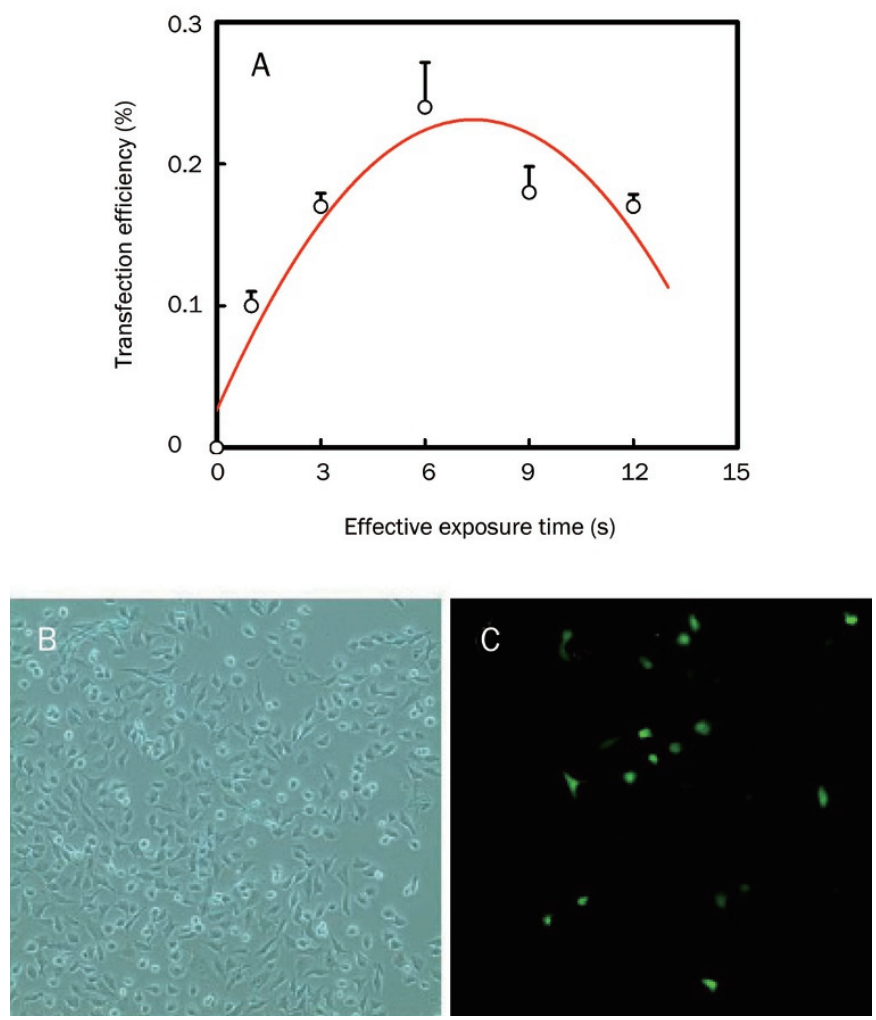

Figure 3. (A) Transfection efficiencies of HUVECs versus various effective ultrasound exposure times in the presence of $2 \mu \mathrm{g} / \mathrm{mL}$ EGFP-N1 plasmid. Each point is the mean of five independent experimental data; (B) Brightfield micrograph of cultured HUVECs; and (C) Fluorescence micrograph of cells showing EGFP expression following ultrasound exposure of $6 \mathrm{~s}$ (magnification $\times 15)$.

increased with the effective exposure time, and then decreased when the exposure time was beyond $6 \mathrm{~s}$. In other words, there was a transfection efficiency peak at $6 \mathrm{~s}$ of exposure (Figure 3A). The increase in transfection efficiencies at the first stage was due to the fact that more plasmid DNA could enter the HUVECs with increased exposure time. However, the number of dead cells was higher than that of transfected cells when the effective exposure time was longer than $6 \mathrm{~s}$. As a result, the transfection efficiencies decreased in the second stage $(>6 \mathrm{~s})$. This phenomenon was also observed in our previous studies on HeLa-S3 cells ${ }^{[24]}$. The number of dead cells and transfection efficiency increased with exposure time. However, cell death or lysis predominated, leading to a decrease in transfection efficiency. Some cells may have successfully incorporated the plasmid following ultrasound sonoporation, but they could not express the transferred gene because they died quickly.

Figure 4 shows the effects of increasing SonoVue microbubble concentrations on the transfection efficiencies of HUVECs. After $6 \mathrm{~s}$ of ultrasound exposure, the transfection efficiencies were quantified by flow cytometry. The transfection efficiencies were relatively high in the presence of $10 \%$ and $20 \%(v / v)$ SonoVue microbubbles, but there was no significant difference observed between the two concentrations. Interestingly, a

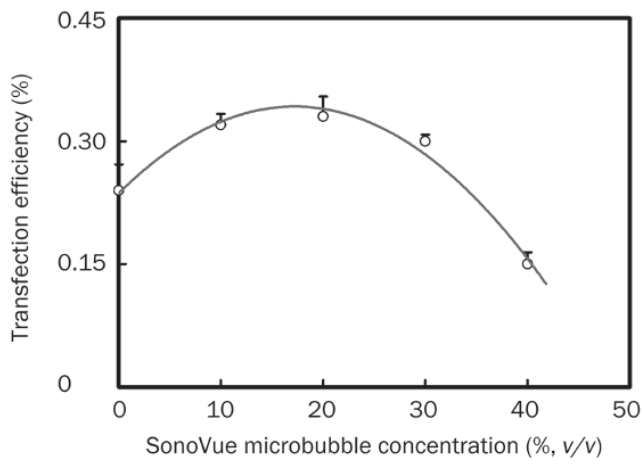

Figure 4. Effect of SonoVue microbubble concentrations on transfection efficiencies of HUVECs following $6 \mathrm{~s}$ of ultrasound exposure with $10 \%$ duty cycle in the presence of $1 \mu \mathrm{g} / \mathrm{mL}$ EGFP-N1 plasmid. The 0\% Sonovue indicates the control of "no SonoVue". The data were obtained from three independent experiments.

high concentration of SonoVue $(40 \%, v / v)$ had no synergistic effect on gene transfection enhancement with ultrasound but caused a further decrease in gene transfection efficiency to levels even lower than those of the control sample $(0 \%$, $v / v)$. One explanation for this phenomenon may be that the high microbubble concentration caused more cell death when combined with the ultrasound field. Thus, we compared the transfection efficiencies of HUVECs in the presence or absence of $10 \%(v / v)$ SonoVue microbubbles. It was found that microbubbles at an appropriate concentration $(10 \%, v / v)$ could significantly enhance cell transfection efficiency (Figure 5). The transfection efficiency of HUVECs in the presence of microbubbles with $6 \mathrm{~s}$ of ultrasound exposure (the peak) was approximately 3-fold higher than in the absence of microbubbles, which also demonstrated that the ultrasound microbubbles had a synergistic effect on gene transfection. However, SonoVue microbubbles are destroyed by ultrasound. This destruction generated high-energy microstreams or microjets that produced shear stress surrounding the cell membranes and increased permeability ${ }^{[11,25]}$. The increase in permeability was probably due to transient "pores" in the plasma mem-

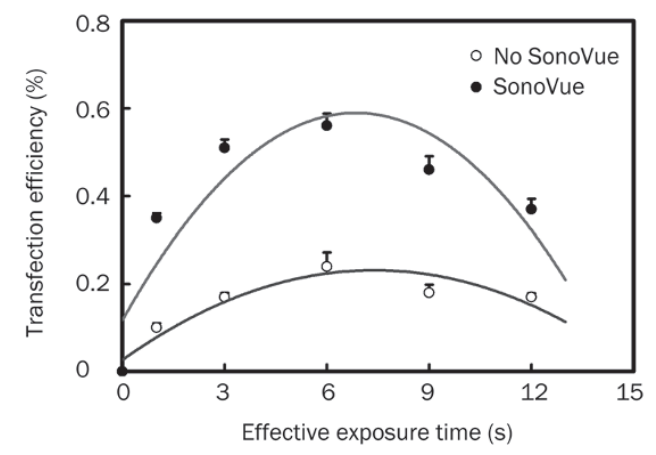

Figure 5. Transfection efficiencies of HUVECs following ultrasound exposure with $10 \%$ duty cycle with or without $10 \%(v / v)$ SonoVue microbubbles in the presence of $1 \mu \mathrm{g} / \mathrm{mL}$ EGFP-N1 plasmid. The data were obtained from three independent experiments. 
brane ${ }^{[26]}$. Ultrasound with microbubbles could potentiate gene delivery by creating transient nonlethal perforations in cell membranes to aid ingress of molecules (DNA or other drugs) into the cells ("sonoporation"). The rapid vibration of microbubbles on the surfaces of the cells, or microstreaming, likely allows for the enhanced delivery of DNA across the cell membrane ${ }^{[11,12,27]}$.

For gene transfections using ultrasound, another important factor affecting efficiency is plasmid concentration. Therefore, the transfection of HUVECs using various concentrations of EGFP-N1 plasmid was also studied. The transfection efficiencies increased with the plasmid DNA concentration, as shown in Figure 6A. This tendency could also be observed in the fluorescent images (Figure 6B). The number of cells that expressed EGFP in the presence of $8 \mu \mathrm{g} / \mathrm{mL}$ of plasmids is significantly high. It is reasonable to assume that there are more chances for plasmid DNA entry into cells if the plasmid concentration is high when the cells are exposed to ultrasound.

The gene transfection data shown in Figures 3-6 reflect transfection efficiencies that are not at the desired levels. In 2008, Wang et al ${ }^{[28]}$ reported that the transient transfection rate of HUVECs was approximately $20.3 \%$ using a recombinant plasmid (pEGFP-KDR-TK) under $1.9 \mathrm{MHz}$ continuous ultrasound with $80 \mathrm{~mW} / \mathrm{cm}^{2}$ output intensity. In our opinion, it is reasonable that different ultrasound exposure setup and exposure modalities can greatly affect gene transfection efficiency. Other parameters, such as exposure intensity, frequency, type of cell culture plate, cell type, and plasmid type and size, will also remarkably affect gene transfection efficiency. Our data showed that the technique of immersing the microtip directly into the cell culture medium was not preferable to the reported methods of Wang et al ${ }^{[28]}$. Further investigation is still needed to reach acceptable levels of gene transfection in HUVEC cultures.

\section{Delivery of model drugs into HUVECs using ultrasound}

Ultrasound can also be used in drug delivery, particularly for cardiovascular disease therapy. Figure 7 shows that TO$\mathrm{PRO}^{\circledR}-1$ molecules can be delivered into HUVECs with high efficiency. TO-PRO ${ }^{\oplus}-1$ is a nucleic fluorescent dye with a low
A

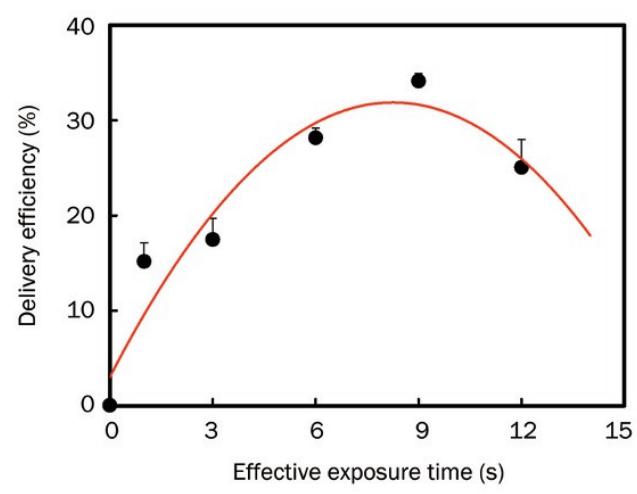

B
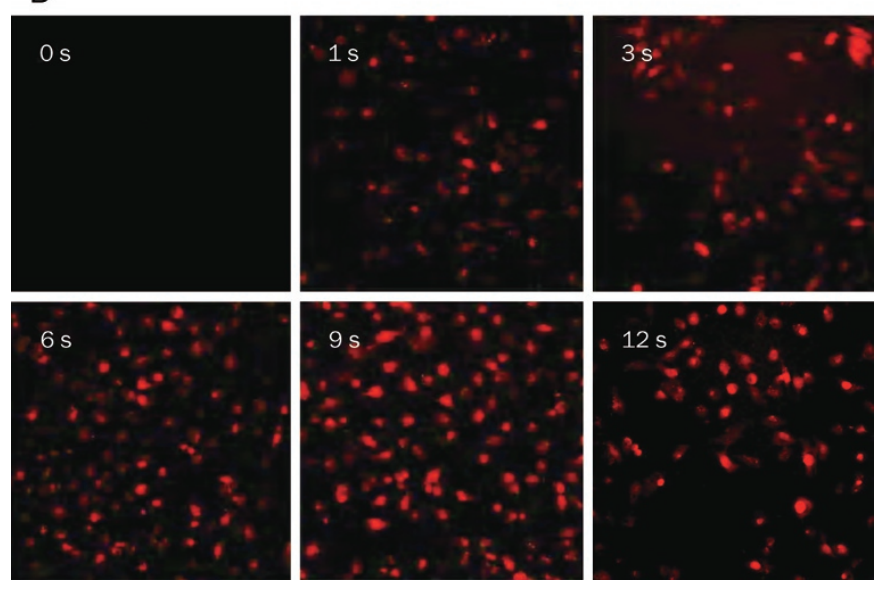

Figure 7. (A) Cellular uptake (delivery efficiency) of fluorescent molecules of TO-PRO ${ }^{\circledR}-1$ (as the low molecular weight model drug) into HUVECs following ultrasound exposure in the presence of $10 \mu \mathrm{g} / \mathrm{mL}$ TO-PRO ${ }^{\circledR}-1$. Each point is the mean of three independent experimental data. (B) Fluorescence micrographs of the model drug delivery into HUVECs (magnification $\times 20$ ).

molecular weight of 645.38 daltons and has the unique property of being unable to permeate intact cell membranes. In this study it was used as a model drug to quantify intracellular uptake into viable cells. The drug delivery efficiency was very similar to the observed efficiency of gene transfer to HUVECs.
A

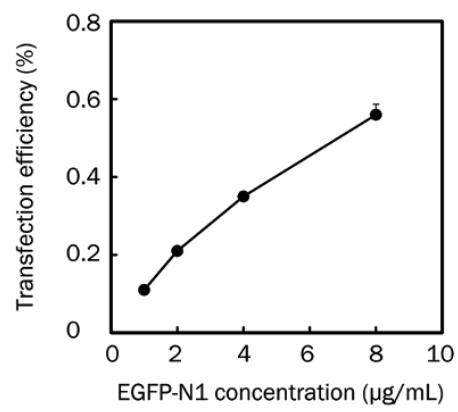

B

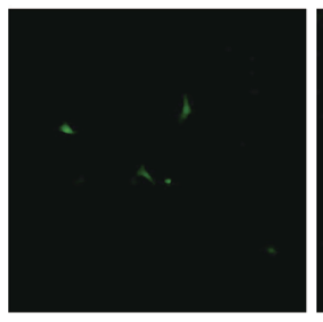

$1 \mu \mathrm{g} / \mathrm{mL}$

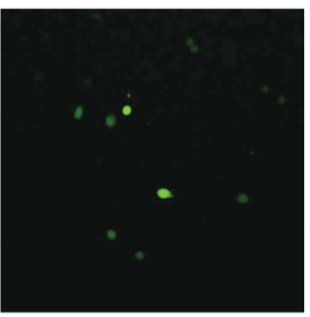

$2 \mu \mathrm{g} / \mathrm{mL}$

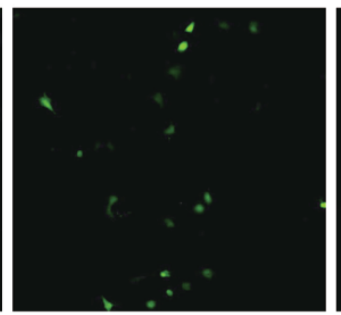

$4 \mu \mathrm{g} / \mathrm{mL}$

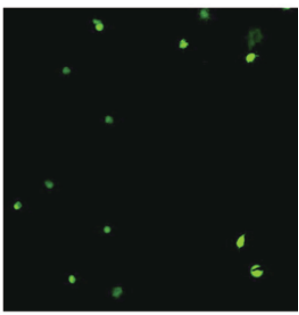

$8 \mu \mathrm{g} / \mathrm{mL}$

Figure 6. (A) Transfection efficiencies versus plasmid concentrations of EGFP-N1 under an effective exposure time of $6 \mathrm{~s}$. Each point is the mean of three independent experimental data. (B) Fluorescence micrographs of EGFP expression in the presence of plasmid at various concentrations (magnification $\times 15$ ). 
However, the peak of delivery was found at an effective exposure time of $9 \mathrm{~s}$, whereas for gene transfer the peak was found at an effective exposure time of $6 \mathrm{~s}$. Notably, the delivery efficiencies of this model drug with a low molecular weight were significantly higher than that of plasmid DNA. The maximum efficiency was approximately $34 \%$-more than 150 -fold that of plasmid DNA transfection. To determine whether this phenomenon was also observed in the case of other drugs, a highmolecular-weight model drug, Dextran Rhodamine B (70000 Dalton), was used in our experiments (Figure 8). Interestingly, the delivery efficiencies were very low, and the maximum transfection efficiency was $0.5 \%$ at an effective exposure time of $15 \mathrm{~s}$, which was very similar to the delivery efficiency of EGFP-N1 plasmid DNA. One possible reason for this result is that small molecules can easily enter the cells following ultrasound exposure, which opens some transient "pores" on the cell membrane, whereas the plasmid DNA and Dextran Rhodamine B are macromolecules that diffuse poorly into the cells $^{[29,30]}$.
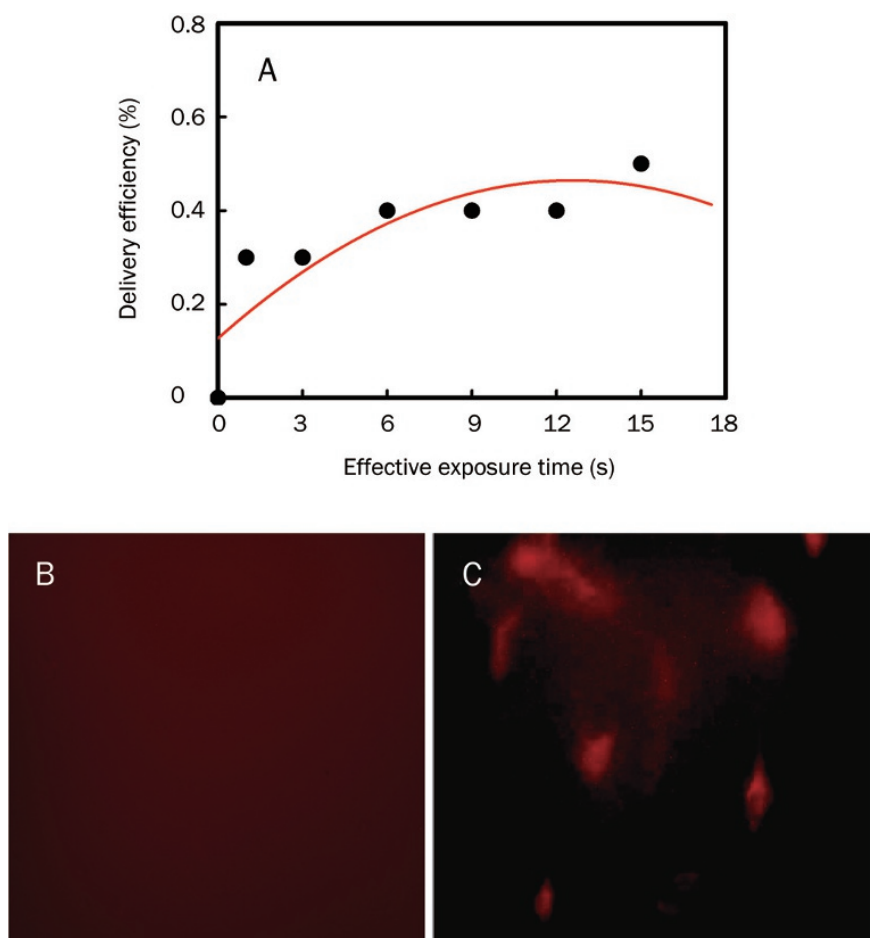

Figure 8. (A) Cellular uptake (delivery efficiency) of fluorescent molecules of Dextran-Rhodamine B (as the high-molecular-weight model drug) into HUVECs following ultrasound exposure in the presence of $40 \mu \mathrm{g} / \mathrm{mL}$ Dextran-Rhodamine B. Each point is the mean of three independent experimental data. Representative fluorescence micrographs showing model drug (Dextran-Rhodamine B) delivery into HUVECs exposed to ultrasound for $12 \mathrm{~s}(\mathrm{C})$ and into control cells (no ultrasound exposure) (B) (magnification $\times 60$ ).

\section{Effects of ultrasound on migration of HUVECs}

To further investigate the biological effects of ultrasound exposure on HUVECs, cell migration ability was assayed in vitro using Millicell ${ }^{\circledR}$ cell culture inserts. Figures 9A and 9B show the transmembrane migration of the control (no exposure) and exposed cells, respectively. It was found that no cell migration occurred during the first $2.5 \mathrm{~h}$, and a few migrated cells were
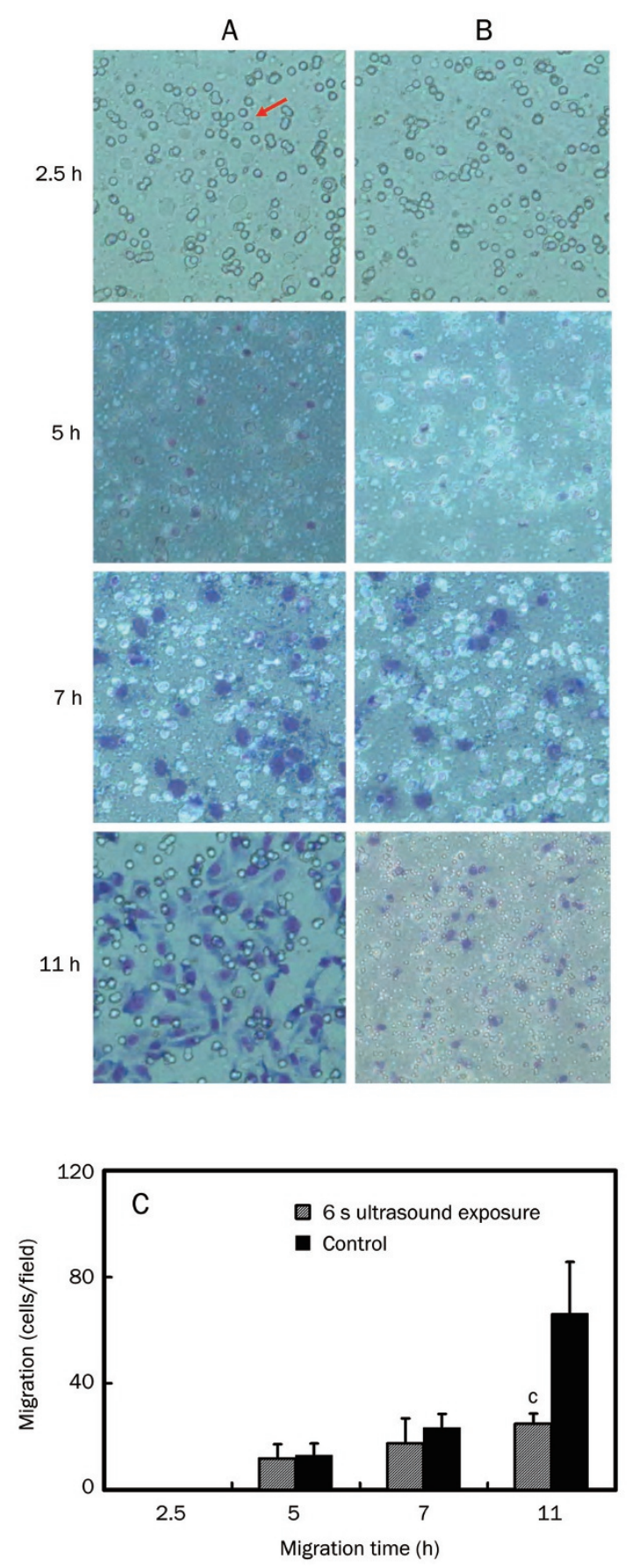

Figure 9. Migration comparison of HUVECs after ultrasound exposure (6 s) (B) and the control (no ultrasound exposure) (A). The cells stained darkblue are the transmembrane-migrated HUVECs. The small open circles (marked by an arrow) are the pores of the PET membrane of Millicell ${ }^{\circledR}$ Cell Culture Inserts (magnification 60x). (C) The numbers of migrated cells were quantified by cell counting from 10 microscopic fields for each culture time point. The data were obtained from three independent experiments. ${ }^{\circ} P<0.01$ vs control at $11 \mathrm{~h}$. 
observed after incubation for $5 \mathrm{~h}$. This lack of migration is due to the time that cells need to adhere to the PET membranes before migration could occur. Many migrated cells (stained dark blue) were observed, particularly after $11 \mathrm{~h}$ of incubation, but the number was still lower than that of the control. It could be concluded that ultrasound exposure decreased HUVEC mobility and migration. Given the importance of the cytoskeleton to cell adhesion and migration, there is a possibility that the cytoskeleton of the cells exposed to ultrasound would be disordered. Aside from the cytoskeleton, it is also possible that the activities of some proteins or enzymes in the cells were also affected by ultrasound. In our previous study, ultrasound exposure was shown to affect some protein activity, for instance, that of $\mathrm{Ca}^{2+}$-ATPase ${ }^{[31]}$. To fully understand the molecular mechanisms and the biological effects of ultrasound, further investigations are necessary.

\section{Summary}

Although the primary effect of ultrasound exposure in vitro is cell lysis induced by ultrasound cavitation, sublethal damage may also occur with the passage of some molecules (eg, DNA and drugs). The transient permeability of cell membranes will lead to the uptake of extracellular molecules into the cells. Our study showed that sonoporation, in the presence of microbubbles, was a promising technique that permits the transfer of drugs and genes into cells. Additionally, low-frequency and low-energy ultrasound-induced destruction of microbubbles is a feasible and efficient method of vascular gene transfection and drug delivery. Although the exact mechanisms underlying efficient drug uptake and gene transfection remain incompletely understood, the rapid collapse of microbubbles during sonoporation has been considered to play a major role in drug/gene delivery into cells. It has also been speculated that the presence of microbubbles can significantly reduce the threshold of acoustic cavitation upon ultrasound exposure in a target site. At this time, the study of the application of microbubbles with ultrasound for gene/drug delivery is still currently in its infancy, particularly in vivo.

\section{Acknowledgements}

This work was supported in part by the National Natural Science Foundation of China (Grant No 30700151), by New Century Excellent Talents Program in Chinese Universities to Yi-yao LIU, by the Innovative Talent Incubation Program for Youth (Grant № Y02018023601062) and by the Starting Fund (Grant No Y02002010901008) from UETSC. We also wish to thank Prof Hong ZHOU for the generous gift of pEGFP-N1, and Prof Zi-li YOU for cell culture assistance and facilities in our department.

\section{Author contribution}

Yi-yao LIU designed the research; Hong YANG, Zhong-hua LIU, and Chang-chun LOU performed research; Zheng-long REN and Hirokazu MIYOSHI contributed new analytical tools and reagents; Hong YANG and Yi-yao LIU wrote the paper.

\section{References}

1 Duvshani-Eshet M, Benny O, Morgenstern A, Machluf M. Therapeutic ultrasound facilitates antiangiogenic gene delivery and inhibits prostate tumor growth. Mol Cancer Ther 2007; 6: 2371-82.

2 Tata DB, Dunn F, Tindall DJ. Selective clinical ultrasound signals mediate differential gene transfer and expression in two human prostate cancer cell lines: LnCap and PC-3. Biochem Biophys Res Commun 1997; 234: 64-7.

3 Teupe C, Richter S, Fisslthaler B, Randriamboavonjy V, Ihling C, Fleming $\mathrm{l}$, et al. Vascular gene transfer of phosphomimetic endothelial nitric oxide synthase (S1177D) using ultrasound-enhanced destruction of plasmid-loaded microbubbles improves vasoreactivity. Circulation 2002; 105: 1104-9.

4 Frenkel V. Ultrasound mediated delivery of drugs and genes to solid tumors. Adv Drug Deliv Rev 2008; 60: 1193-208.

5 Stieger SM, Caskey CF, Adamson RH, Qin S, Curry FR, Wisner ER, et al. Enhancement of vascular permeability with low-frequency contrastenhanced ultrasound in the chorioallantoic membrane model. Radiology 2007; 243: 112-21.

6 Iwanaga K, Tominaga K, Yamamoto K, Habu M, Maeda H, Akifusa S, et al. Local delivery system of cytotoxic agents to tumors by focused sonoporation. Cancer Gene Ther 2007; 14: 354-63.

7 Wu J, Pepe J, Rincon M. Sonoporation, anti-cancer drug and antibody delivery using ultrasound. Ultrasonics 2006; 44: e21-5.

8 Shohet RV, Grayburn PA. Potential bioeffects of ultrasonic destruction of microbubble contrast agents. J Am Coll Cardiol 2006; 47: 1469-70.

9 Taniyama Y, Tachibana K, Hiraoka K, Namba T, Yamasaki K, Hashiya $\mathrm{N}$, et al. Local delivery of plasmid DNA into rat carotid artery using ultrasound. Circulation 2002; 105: 1233-9.

10 Unger EC, Hersh E, Vannan M, McCreery T. Gene delivery using ultrasound contrast agents. Echocardiography 2001; 18: 355-61.

11 Liu Y, Miyoshi H, Nakamura M. Encapsulated ultrasound microbubbles: therapeutic application in drug/gene delivery. J Control Release 2006; 114: 89-99.

12 Liu Y, Yang H, Sakanishi A. Ultrasound: mechanical gene transfer into plant cells by sonoporation. Biotechnol Adv 2006; 24: 1-16.

13 Ohta S, Suzuki K, Tachibana K, Yamada G. Microbubble-enhanced sonoporation: efficient gene transduction technique for chick embryos. Genesis 2003; 37: 91-101.

14 Aschkenasy C, Kost J. On-demand release by ultrasound from osmotically swollen hydrophobic matrices. J Control Release 2005; 110: 58-66.

$15 \mathrm{Kim} \mathrm{H}-\mathrm{J}$, Matsuda H, Zhou H, Honma I. Ultrasound-triggered smart drug release from a poly(dimethylsiloxane)-mesoporous silica composite. Adv Mater 2006; 18: 3083-8.

16 Siu T, Jackson J, Burt H, Chiao M. Drug uptake enhancement using sonodynamic effects at $4 \mathrm{MHz}$ - a potential application for microultrasonic-Transducers. IEEE Trans Biomed Eng 2007; 54: 1153-6.

17 van Wamel A, Kooiman K, Harteveld M, Emmer M, ten Cate FJ, Versluis $\mathrm{M}$, et al. Vibrating microbubbles poking individual cells: drug transfer into cells via sonoporation. J Control Release 2006; 112: 149-55.

18 Liu Y, Yang H, Otaka K, Takatsuki H, Sakanishi A. Effects of vascular endothelial growth factor (VEGF) and chondroitin sulfate $A$ on human monocytic THP-1 cell migration. Colloids Surf B Biointerfaces 2005; 43: $216-20$.

19 Kawai N, lino M. Molecular damage to membrane proteins induced by ultrasound. Ultrasound Med Biol 2003; 29: 609-14.

20 Marentis TC, Kusler B, Yaralioglu GG, Liu S, Haeggstrom EO, Khuri- 
Yakub BT. Microfluidic sonicator for real-time disruption of eukaryotic cells and bacterial spores for DNA analysis. Ultrasound Med Biol 2005; 31: 1265-77.

21 Miller DL, Averkiou MA, Brayman AA, Everbach EC, Holland CK, Wible $\mathrm{JH} \mathrm{Jr}$, et al. Bioeffects considerations for diagnostic ultrasound contrast agents. J Ultrasound Med 2008; 27: 611-32; quiz 633-6.

22 Skyba DM, Price RJ, Linka AZ, Skalak TC, Kaul S. Direct in vivo visualization of intravascular destruction of microbubbles by ultrasound and its local effects on tissue. Circulation 1998; 98: 290-3.

23 Takatsuki H, Furukawa T, Liu Y, Hirano K, Yoshikoshi A, Sakanishi A. Effect of contrast media on vascular smooth muscle cells. Acta Radiol 2004; 45: 635-40.

24 Liu Y, Uno H, Takatsuki H, Hirano M, Sakanishi A. Interrelation between HeLa-S3 cell transfection and hemolysis in red blood cell suspension using pulsed ultrasound of various duty cycles. Eur Biophys J 2005; 34: 163-9.

25 Bao S, Thrall BD, Miller DL. Transfection of a reporter plasmid into cultured cells by sonoporation in vitro. Ultrasound Med Biol 1997; 23: 953-9.
26 Deshpande MC, Prausnitz MR. Synergistic effect of ultrasound and PEI on DNA transfection in vitro. J Control Release 2007; 118: 126-35.

27 Tachibana K, Uchida T, Ogawa K, Yamashita N, Tamura K. Induction of cell-membrane porosity by ultrasound. Lancet 1999; 353: 1409.

28 Wang Y, Xu HX, Lu MD, Tang Q. Expression of thymidine kinase mediated by a novel non-viral delivery system under the control of vascular endothelial growth factor receptor 2 promoter selectively kills human umbilical vein endothelial cells. World J Gastroenterol 2008; 14: 224-30.

29 Mehier-Humbert S, Bettinger T, Yan F, Guy RH. Ultrasound-mediated gene delivery: kinetics of plasmid internalization and gene expression. J Control Release 2005; 104: 203-11.

30 Rosenthal I, Sostaric JZ, Riesz P. Sonodynamic therapy - a review of the synergistic effects of drugs and ultrasound. Ultrason Sonochem 2004; 11: 349-63.

31 Liu Y, Yang H, Takatsuki H, Sakanishi A. Effect of ultrasonic exposure on $\mathrm{Ca}^{2+}$-ATPase activity in plasma membrane from Aloe arborescens callus cells. Ultrason Sonochem 2006; 13: 232-6.

\title{
5th International Workshop on Vaccine Adjuvants and Parasitic Vaccines 1st Symposium of Nano and Micro Particles. ADJUVANT 2010
}

\author{
Trinidad, Sancti Spíritus, Cuba \\ 16-21 May 2010 \\ Contact: Dr Judith del Campo \\ E-mail: adjuvant@finlay.edu.cu
}

